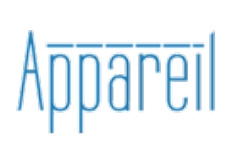

Appareil

$23 \mid 2021$

Poïétique du jeu vidéo

\title{
Des relations entre jouer et créer des jeux vidéo
}

La piste de l'improvisation au prisme d'une approche pragmatique

Julie Delbouille et Pierre-Yves Hurel

\section{CpenEdition}

Journals

\section{Édition électronique}

URL : http://journals.openedition.org/appareil/3921

DOI : 10.4000/appareil.3921

ISSN : 2101-0714

Éditeur

MSH Paris Nord

Référence électronique

Julie Delbouille et Pierre-Yves Hurel, « Des relations entre jouer et créer des jeux vidéo », Appareil [En

ligne], 23 | 2021, mis en ligne le 31 mars 2021, consulté le 02 avril 2021. URL : http://

journals.openedition.org/appareil/3921 ; DOI : https://doi.org/10.4000/appareil.3921

Ce document a été généré automatiquement le 2 avril 2021.

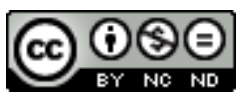

Appareil est mis à disposition selon les termes de la Licence Creative Commons Attribution - Pas d'Utilisation Commerciale - Pas de Modification 4.0 International. 


\section{Des relations entre jouer et créer des jeux vidéo}

La piste de l'improvisation au prisme d'une approche pragmatique

Julie Delbouille et Pierre-Yves Hurel

\section{Introduction}

1 La production de jeu vidéo est régulièrement décrite, analysée et décortiquée à l'aune de ses processus industriels (comme l'ont déjà souligné Julien Rueff ${ }^{1}$ ou Vinciane Zabban ${ }^{2}$ ). Parallèlement, un champ d'étude portant sur l'objet-jeu et l'expérience ludique, dont les problématiques délaissent souvent les questions de production, s'est développé au cours des dernières décennies - avec l'émergence, audelà des publications issues du game design, des approches narratologiques ${ }^{3}$ et formelles ${ }^{4} \mathrm{du}$ jeu, ou encore des études ethnographiques ou sociologiques de ses pratiques $^{5}$. Ce faisant, les études du jeu (game studies) tendent souvent à dissocier d'une part, le processus de création (qui serait entièrement régulé par les contraintes techniques, économiques, etc.) et, d'autre part, l'objet produit (le jeu ou le game) et les pratiques ludiques qu'il orchestre (les plays). Dans cet article, notre objectif est de faire dialoguer les activités «créer » et "jouer », afin de proposer une réflexion théorique sur les liens qui les unissent. Si ce rapprochement entre jouer et créer peut sembler intuitif, il reste cependant difficile de saisir en quoi ces notions se recoupent : étudier l'articulation entre ces deux activités nous permettra, en outre, d'éclairer leurs spécificités propres.

2 Cette étude des liens entre jouer et créer un jeu vidéo est le fruit d'un dialogue entre les enquêtes de terrain de nos recherches doctorales respectives. D'une part, les travaux de Julie Delbouille ${ }^{6}$, qui se focalisent sur l'avatar et le personnage vidéoludique, ont pu mettre en évidence la dimension créative du jouer dans le cadre de l'appropriation par les joueurs d'une entité vidéoludique. Afin de prolonger son expérience de jeu, le joueur doit sans cesse puiser dans les éléments déjà intégrés à son répertoire ludique et improviser de nouvelles associations. D'autre part, la thèse de Pierre-Yves Hurel ${ }^{7}$ 
propose de comprendre la création de jeux vidéo en amateur comme un processus reposant en grande partie sur l'improvisation de ses auteurs. Ces derniers prennent des dispositions (par des recherches et apprentissages préalables) pour que leur pratique de création consiste avant tout en la gestion d'incertitudes, qui revêtent une dimension ludique: il s'agit de se placer comme premier public de la création en cours, de s'interroger sur la satisfaction qu'elle produit ou non et de se laisser surprendre par des résultats inattendus. La mise en dialogue de ces recherches a été d'autant plus opérante, que leur perspective méthodologique repose sur une approche commune. Chaque enquête articule une série d'entretiens compréhensifs ${ }^{8}$ analysés sur le plan qualitatif (s'inspirant notamment des travaux de Christophe Lejeune ${ }^{9}$ ): elles construisent ainsi un échantillon de dix-huit joueurs (interrogés par Delbouille de 2016 à 2019) et de vingt-deux créateurs amateurs ${ }^{10}$ (questionnés par Hurel de 2015 à 2019). Enfin, il se trouve que les personnes recrutées dans le cadre de chaque enquête sont particulièrement impliquées dans le jeu et/ou dans la création : les résultats présentés dans cet article ont pour point de départ l'analyse de pratiques de ce qu'on pourrait qualifier de "grands amateurs » de jeu vidéo - cette expression faisant ici référence non à une compétence réputée, mais bien à un engagement marqué dans ces activités.

La mise en commun de ces deux terrains a permis de faire émerger une notion comme point de jonction entre le jeu et la démarche créative : l'improvisation. Lorsque nos informateurs ${ }^{11}$ évoquent la dimension ludique de la création, ainsi que la dimension créative dans le jeu, le dénominateur commun est en effet identique : la rencontre entre jeu et création, dans leur propre expérience, se situe dans le geste improvisé. Entendue ici dans son acception la plus commune, l'improvisation consiste, pour les joueurs comme les créateurs de jeux vidéo, à faire coïncider conception et réalisation et ainsi à produire un résultat non planifié - un play nouveau pour le joueur, une séquence d'œuvre inédite pour le créateur amateur -, notamment en puisant librement dans les connaissances et compétences préalablement acquises. Ces dernières constituent une forme de "répertoire ludique " composé de séquences, de mécaniques, de tropes accumulés au cours des différentes expériences de jeu, répertoire au sein duquel le joueur et le créateur sélectionnent des éléments pour produire de nouvelles associations. Comme le souligne Serge Margel, la préparation de l'improvisation comporte tant la constitution de ce répertoire que la mise en place des conditions propices à sa mobilisation : «En ce sens, la préparation ne représente ni une simple réserve d'opérations minimales, que l'improvisateur pourrait saisir au bon moment, ni une pure contrainte formelle, comme une compétence instrumentale, la maîtrise des règles du jeu ou la connaissance des codes culturels, mais c'est d'abord et avant tout l'organisation d'un plan ou du cadre interactionnel de l'action ${ }^{12}[. .$.$] ». Ainsi, improviser$ revient à construire et à éprouver un cadre qui permet de "trouver des astuces pour avancer, pour continuer», d'inventer «sur-le-champ», "franchir l'obstacle, contourner l'accident, retrouver sa trajectoire, son chemin, son geste, son rythme ${ }^{13}$ [...] ». Central dans les expériences vidéoludiques de nos informateurs, ce recours à l'improvisation y est également indissociable de la dimension créative associée au jeu, comme le soulignait également Donald Winnicott ${ }^{14}$ : dans ce contexte, la créativité du joueur ou du créateur de jeux vidéo est entendue comme sa faculté à produire des associations inédites, à se jouer de l'inattendu ou encore à recourir à des solutions originales - dans une acception plus large que celle qui lui est traditionnellement associée dans le domaine artistique. 
4 L'articulation de nos recherches a également fait apparaître la nécessité de recourir à une autre notion particulièrement opératoire : celle de "prise ». L'usage que nous en ferons est issu d'une étude pragmatique de Christian Bessy et Francis Chateauraynaud ${ }^{15}$ sur les experts et les faussaires, où la notion de "prise » désigne ce qui permet de maintenir la relation entre, d'une part, les conventions sur lesquelles nous appuyons notre jugement à propos d'un ou plusieurs objets et, d'autre part, le résultat de notre corps-à-corps avec ces mêmes objets (qui «se plient ou ne se plient pas à des formes d'action ou d'interprétation $\left.{ }^{16} »\right)$. Ainsi, le buraliste développe une certaine manière de prendre un billet de banque, lorsqu'il tente de détecter un faux : il le palpe et le passe sous ses doigts pour saisir ce que ce corps-à-corps lui renvoie en termes d'impressions et de sensations. Dans le contexte du jeu vidéo, la recherche de ce corps-à-corps passe donc d'abord par la situation de jeu elle-même : ce jeu spécifique, qui est de l'ordre de tel genre vidéoludique, possède telle caractéristique singulière - reconnue, ou inédite dans l'expérience du joueur. Dans le cas de l'amateur de jeu vidéo, qui est guidé par sa recherche d'effets (et non par une quête de l'authentification de l'objet, comme chez Bessy et Chateauraynaud), le développement d'une prise est mis au service de ses attachements, de ses préférences, de ses goûts en matière de jeu. Dès lors, ce qui différencie le néophyte du joueur ou du créateur amateur est un savoir-prendre spécifique de l'objet - qui se développe tant par la dimension ludique que créative de son expérience, comme nous le verrons plus loin.

5 En s'appuyant sur ces différentes notions, notre argumentation est structurée en trois étapes. Premièrement, nous identifions le rôle de l'improvisation dans le "jouer » (au sens de Jacques Henriot, 1989), en tentant ainsi de saisir sa part créative. Deuxièmement, nous rendons compte de l'importance de la dynamique d'improvisation propre à la création de jeux vidéo amateurs. Enfin, dans un troisième temps, nous cherchons à comprendre les liens théoriques unissant « créer » et « jouer » et mettons finalement en lumière l'interdépendance qu'elles entretiennent dans les contextes étudiés.

\section{Inventer et réinventer le play: le double rôle de l'improvisation dans le jouer}

\section{Incorporer les codes du jeu}

Contrairement au présupposé commun sur le caractère « automatique » de l'expérience vidéoludique, la prise en main du jeu vidéo est loin d'être naturelle. D'une part, elle est le résultat de toute une série de processus d'apprentissage, qui s'appuient non seulement sur l'expérience immédiate du joueur, mais aussi, plus largement, sur sa culture vidéoludique et son " capital ludique ${ }^{17}$ » - désignant l'ensemble des références, codes, réflexes et gestes précédemment acquis par le joueur. D'autre part, cette prise en main est bardée de références et de conventions qui, si elles cherchent à se faire oublier par leur récurrence au sein du médium, nécessitent néanmoins une première intégration dans le répertoire du joueur. La manipulation d'un jeu (qu'elle passe par la prise en main d'un corps virtuel, d'un curseur ou autre) manifeste constamment cette nécessaire phase d'incorporation : le joueur s'approprie un geste, un signe, un code qui est au départ nouveau, étranger ${ }^{18}$. Ils entrent ensuite, progressivement, dans son répertoire ludique, deviennent conventionnels et acquièrent un sens propre qui n'est 
ensuite plus questionné ou réfléchi ; ce processus se répète tout au long de l'expérience ludique. Les errances des premiers gestes posés ou la prise en main parfois balbutiante de l'environnement virtuel sont régulièrement convoqués dans le discours de nos informateurs. L'un d'entre eux, Gauthier, évoque ainsi sa relation aux premiers instants de jeu et, plus spécifiquement, aux didacticiels qu'ils comportent souvent :

Alors, je fais partie des gens qui détestent les didacticiels [rires] et qui préfèrent lancer une partie pour du beurre, où ils se font péter la gueule quarante fois, et qui se disent, bon, je vais quand même peut-être aller voir le didacticiel... [...] Donc c'est ça, c'est le début, et alors j'ai une forte tendance à toujours recommencer quatre, cinq fois le début du jeu. [...] C'est vraiment essayer de voir toutes les possibilités du jeu effectivement [...].

Comme l'illustre cet extrait d'entretien, la prise en main du jeu s'inscrit souvent dans une phase de tâtonnements. L'un des objectifs des didacticiels est a priori de donner au joueur une prise sur les instruments garants de son agentivité au sein de l'univers de jeu. Ce guide d'apprentissage prend des formes multiples: passage obligé ou étape " dispensable ", il peut être séparé du jeu proprement dit ou intégré à la diégèse, voire à la narration. Apprendre à marcher, courir, sauter, à saisir les objets, à utiliser un inventaire, à interagir et à dialoguer avec l'environnement et les personnages nonjoueurs, mais aussi plus largement à comprendre les codes élémentaires qui régissent le jeu : les pré-requis exposés dans le tutoriel varient en fonction de l'œuvre concernée, mais témoignent de l'existence d'un ensemble (même minimal) de mécaniques que le joueur doit apprendre à maîtriser pour accéder à une première expérience possible du jeu.

8 Que ce soit au sein d'un didacticiel ou, comme dans le cas de Gauthier, en évitant ce dernier, ce rôle d'acquisition des contrôles, mécaniques et règles de base du jeu endossé par les guides d'apprentissage fait apparaitre une caractéristique récurrente de ces premiers moments de jeu: le tâtonnement, l'errance, la découverte par essai-erreur. Dans certains cas, le joueur doit donc mobiliser les outils qui lui permettent d'agir dans le jeu avant de les avoir entièrement pris en main - voire avant que le jeu ne lui ait suggéré cet embryon de " jeu-modèle » transmis par le didacticiel (et que le joueur peut volontairement décider d'ignorer).

9 Ces phases d'apprentissage, qui peuvent se répéter tout au long du jeu, sont le lieu de nombreuses improvisations de la part du joueur : celles-ci visent, dans ce cas, à tenter de progresser, tout en appréhendant les possibles du jeu. Cette improvisation entre en tension avec le play modèle que tente de suggérer le jeu, et qui passe par de nombreux signaux visuels, auditifs ou liés aux mécaniques de jeu - comme le fait de ne pas donner accès à la suite d'un niveau tant qu'une séquence spécifique n'a pas été réalisée par le joueur. Plusieurs informateurs témoignent d'ailleurs de leurs tactiques pour reprendre le contrôle sur cette expérience d'appropriation du jeu, en ignorant par exemple certains inputs de la structure ludique, comme l'exprime cet autre informateur, Alexandre :

Mais ça c'est parce que j'ai besoin vraiment de la liberté, j'ai besoin d'acquérir les bases et de me dire c'est bon, après cinq ou dix minutes, vous me laissez faire ce que j'ai envie maintenant, tu vois.

10 Dans cette phase d'appropriation, le rôle traditionnellement dévolu à l'objet-jeu est donc de lever le voile sur sa structure, ses règles, ses codes et de les rendre suffisamment accessibles (même si cette accessibilité peut rester lacunaire, fragmentée, minimale), afin de donner au joueur une prise sur le jeu, mais aussi de lui laisser la 
possibilité d'expérimenter par lui-même les possibles ludiques en improvisant. Ce sont alors les limites du jeu que les joueurs essayent d'atteindre : « est-ce qu'il m'est possible de faire ça ?", « et si j'effectue tel geste, à tel moment, que va-t-il se passer ? ", « est-ce que je peux passer par ici?", etc. Cette improvisation se distingue des pratiques d'exploration (voir, à ce sujet, les travaux de Guillaume Grandjean ${ }^{19}$ ), qui relèvent d'une découverte spatialisée du jeu. En effet, alors que ces dernières s'inscrivent prioritairement dans le domaine intradiégétique (se mêlant à la narration, ou à tout le moins, à l'univers fictionnel du jeu), l'improvisation que nous évoquons est d'origine extradiégétique : elle touche en effet à la mobilisation du répertoire d'actions du joueur et à un assemblage sans cesse renouvelé des éléments qui le composent. Si cette mobilisation a bien un effet sur la diégèse du jeu (telle séquence de gestes effectuée par le joueur aura tel impact sur l'environnement que son avatar est en train de parcourir), elle est formée à l'extérieur de celui-ci par le joueur en réponse à la structure de jeu. Ce recours à l'improvisation par les joueurs vise, dans ce contexte, à développer leur prise propre sur le jeu, avec ou au-delà de celle que leur offre le game. En tentant d'atteindre les frontières du jeu, de soulever par lui-même le coin du voile, le joueur peut ainsi mettre en place une autre tactique d'appropriation, une appropriation par la limite qui tente même parfois de «casser le jeu » (pour reprendre les propos d'un de nos informateurs).

\section{Mobiliser son répertoire ludique pour réinventer le play}

À cette première forme d'improvisation, intimement liée à l'apprentissage et à la mise à l'épreuve des possibles du jeu, s'en superpose une seconde dans le discours des informateurs : celle qui permet non plus d'inventer un play, mais de le réinventer. En effet, lorsque le joueur s'est approprié les instruments et les codes du jeu, lorsque ces derniers sont lexicalisés et inscrits dans le répertoire d'action du joueur, il atteint un moment de statu quo. Ce moment de jeu spécifique déclenche chez nos informateurs une expérience double. Ils prennent d'abord du plaisir à déployer les compétences nouvellement acquises, à affiner leur maîtrise des instruments de jeu; les médiations semblent soudainement "s'effacer", au profit d'une prise ressentie comme plus directe, immédiate, sur l'environnement ludique. Le joueur fait ainsi corps avec le jeu. Cependant, lorsque cet état de grâce se prolonge, le plaisir de jeu se dilue, l'intérêt s'étiole, le sentiment de progression se perd : lorsque chaque geste se fait l'énième écho d'un geste antérieur, comme une partition sans cesse rejouée, il révèle le caractère artificiel de la structure du jeu. Si ce statu quo persiste, le jeu s'épuise. C'est pourquoi la plupart des jeux multiplient les phases d'appropriation: lorsque le joueur atteint un nouveau palier dans son apprentissage, une nouvelle mécanique, une nouvelle compétence, une nouvelle quête est introduite.

Plus le joueur maîtrise le jeu, ses codes, ses mécaniques, plus le résultat de ses actions dans l'environnement du jeu est certain: le déroulement du jeu ne surprend plus, laissant apparaître le squelette, la structure initiale, devenue évidente, de l'œuvre. Seule l'amorce d'un nouveau processus d'appropriation permet de "remettre du jeu » dans le jeu, en réintroduisant dans le play désormais prévisible une forme d'incertitude ludique. Henriot faisait déjà de cette incertitude l'une des conditions sine qua non de l'existence du jeu : « jouer, c'est ne pas savoir où l'on va, même si l'on a soigneusement préparé son itinéraire et calculé ses effets ${ }^{20} »$. Comme le reformule Maude Bonenfant, «tout jeu comprend une marge d'incertitude et d'indétermination, non pas 
nécessairement au niveau de l'objet-jeu, mais pour le sujet-joueur qui ignore l'ensemble des causes ${ }^{21} »$. Cette incertitude constitue un des moteurs essentiels du jeu : ce dernier n'existe « qu'à partir du moment où un joueur s'aventure dans l'imprévisible et y risque quelque chose ${ }^{22} »$.

Réintroduire de l'incertitude permet donc de rendre à l'objet-jeu, à la structure, au game, son potentiel ludique. De plus, si cette manœuvre peut être opérée par le jeu luimême (en proposant, par exemple, une nouvelle mécanique de gameplay que le joueur n'avait pas anticipée, une nouvelle compétence à acquérir, etc.), le joueur peut également réintroduire, par ses propres actions, du jeu dans les rouages désormais trop serrés de la machine ludique. C'est ici que l'improvisation intervient : elle vise alors non plus seulement à jouer, mais à jouer autrement, à investiguer d'autres plays possibles. Le même passage, la même quête, le même combat, bien que déjà actualisé, sont remis en jeu: dans l'expérience des joueurs, la volonté de réussir ou de progresser est supplantée par celle de découvrir ou de se dépasser.

Ce second ensemble d'improvisations, comme le premier, comporte nécessairement une part créative : l'improvisation vise non seulement à produire un play " pertinent ", mais à en créer d'autres modalités, à ressentir le jeu autrement. L'objet du plaisir ludique, qui jusqu'alors résidait dans l'atteinte de l'objectif, se déplace : l'objectif doit désormais être atteint avec "originalité ", avec "spectacularité », ou encore avec "style ${ }^{23}$ "-quitte à recommencer inlassablement les mêmes moments de jeu en improvisant à chaque fois de nouvelles combinaisons de choix ou de gestes. Cette démarche de création de nouvelles expériences ludiques ${ }^{24} s^{\prime}$ inscrit dans une forme de performance, au cours de laquelle le joueur déploie le répertoire d'actions précédemment assimilé lors des phases d'apprentissage (qu'il s'agisse du répertoire propre au jeu en cours d'utilisation, ou de celui construit au cours de son parcours de joueur). Cette improvisation créative au sein du jeu entretient, comme nous le verrons au point suivant, des parallèles structurels avec l'improvisation dans la création de jeux - et en particulier la double posture de joueur "en train de jouer» et de créateur mesurant et modulant les effets de ses inputs.

\section{La part de jeu dans le créer}

Pour saisir la part du jouer dans la création, il nous faut d'abord préciser que notre enquête a démontré que la conception de jeux vidéo en amateur est vécue par ses acteurs comme un prolongement de l'activité de jeu. La création est, pour eux, un moyen de développer une autre prise sur leur goût pour le jeu vidéo ${ }^{25}$. De plus, elle peut aussi être ressentie, chez nos informateurs, comme une forme de jeu en elle-même, qui ne s'explique pas uniquement par la question du prolongement. Nous allons tenter de comprendre à quelles conditions créer peut être un jeu - et où se situe ce jouer particulier -, en explorant deux aspects de notre objet d'étude : le cheminement des créateurs et les projets créés.

\section{Trajectoire des acteurs : faire ses gammes et stabiliser sa pratique}

Les créateurs de jeux vidéo en amateur que nous avons interrogés ${ }^{26}$ sont des créateurs de longue date qui ont développé des compétences importantes. Ils n'identifient pas de période d'apprentissage qui serait distincte du reste de leur trajectoire. Leur démarche 
ne consiste pas à consulter des ouvrages de référence pour étudier des notions d'informatique générale, puis le fonctionnement du programme, avant de réaliser enfin divers exercices et de commencer à créer. Au contraire, ils effectuent des apprentissages opportunément, en fonction de ce qu'ils souhaitent réaliser - ce qui les distingue, par exemple, des étudiants en programmation. Baptiste explique passer régulièrement de la lecture de tutoriels à la participation à un forum pour poser des questions, ou encore "ouvrir les jeux que j'aimais bien ${ }^{27}$ ». Émile, qui a " horreur des $\mathrm{RPG}^{28}$ où tous les personnages fusionnent avec le héros " sur la carte du monde, a consulté les fichiers d'un jeu dans lequel les personnages se suivent en file indienne, de manière à en " comprendre le fonctionnement " et à être ainsi capable de reproduire le mécanisme. Nos informateurs effectuent donc leur apprentissage non pour le plaisir d'apprendre, mais pour atteindre un but: la (re)production d'une fonctionnalité. Gaspard utilise le terme "apprivoiser " et souligne son but final de produire des œuvres qui lui « ressemblent» :

En gros le logiciel [RPG Maker] est vraiment prévu pour créer des RPG classiques, du coup c'est ce qu'on fait au début, mais en commençant à l'apprivoiser je pense que je me suis mis à faire davantage des trucs qui me ressemblent (c'est-à-dire, davantage mettre en scène des histoires, expérimenter des trucs).

Pour ces acteurs, il ne s'agit pas tant de maîtriser l'ensemble des fonctionnalités de l'outil et d'être prêt à répondre aux futures demandes, que de développer une zone de confort qui leur permet, une fois l'outil "apprivoisé », de créer des jeux qui disent quelque chose de leurs plaisirs et de leurs représentations. Aussi, lorsqu'il entreprend de créer un nouveau jeu, l'amateur ouvre son logiciel de création et agit comme il l'entend : il place son avatar dans un embryon de monde, il dispose ici un adversaire, là, une scène de dialogue, ailleurs, des objets dissimulés et ainsi de suite. Au fur et à mesure de ses actes, il s'interroge sur la satisfaction que cela lui procure, teste son agencement en situation de jeu, modifie certains placements et reprend sa création. Il peut alors s'agir pour les créateurs de tenter de reproduire fidèlement les mécanismes ludiques qui leur plaisent, ou de mieux les exploiter que dans les œuvres d'origine (par exemple, Norman a comme projet d'ajouter un grappin dans son jeu, car il trouve cette fonctionnalité trop peu utilisée ${ }^{29}$ ).

Lorsque les acteurs rencontrent des difficultés techniques, ces dernières stoppent nécessairement l'improvisation au profit de la résolution de problème. Ainsi, les amateurs alternent des phases fluides et sans difficulté et des "moments un peu laborieux ", tel que l'explique Baptiste, comme le fait de " détourer les monstres parce que tu le fais au pixel par pixel dans Photoshop [...] et c'est un peu pénible, mais bon ça te prend une demi-heure et puis voilà, après t'es content parce que t'as un nouveau monstre ». Une fois le nouveau dessin détouré et intégré au logiciel, Baptiste peut reprendre l'improvisation là où il l'avait laissée.

Nos informateurs ont donc besoin de s'assurer une certaine fluidité dans l'acte de création s'ils veulent être en mesure de déployer une improvisation - et non pas uniquement une pensée technique dont le ressort est la résolution de problèmes plus ou moins fastidieux. L'apprentissage technique doit être pensé comme un préalable pour que puisse advenir l'improvisation. La relation à l'outil créatif et le confort de son usage, sa connaissance fine (par exemple, l'incorporation de raccourcis clavier, la familiarité des menus, etc.), conditionnent la possibilité d'improviser. Tout comme dans le domaine musical, «l'acquisition d'une excellente technique instrumentale est indispensable pour avoir sous la main les moyens d'être expressif ${ }^{30} »$. Les amateurs, par 
leur apprentissage, ont développé la maitrise d'une technique instrumentale qui leur permet d'expérimenter (au moins momentanément) un sentiment de création fluide, facile, sans encombre technique - et donc d'improviser.

\section{Trajectoire des projets : maintenir les dispositions nécessaires à l'improvisation}

L'improvisation caractérise fortement le début du développement de chaque nouveau projet amateur. C'est lors de cette étape qu'elle est la plus visible, sans doute parce qu'elle est alors la plus libre. Improviser ne signifie pas faire n'importe quoi, de n'importe quelle manière. Face à la page blanche, l'amateur se demande que faire. Nos informateurs, pour répondre à cette question, partent d'une "vague idée ", à l'image de Baptiste :

J'avais une vague idée [...] donc j'improvisais tout le temps, enfin pas tout le temps, mais le gros de ce que j'ai fait c'était de l'improvisation dans les dialogues et tout ça.

J'ai rien écrit à l'avance.

21 Les praticiens suivent donc une première impulsion: par exemple l'envie de faire interagir deux personnages dans un RPG, ou encore celle de retrouver la sensation spécifique du maniement d'un véhicule anti-gravité. Cette situation particulière de jeu, aussi anecdotique puisse-t-elle paraître, entraîne avec elle toute une série d'associations typiques de genres vidéoludiques et d'habitudes de jeu: imaginer un dialogue dans un RPG demande ainsi de penser la carte du monde où les personnages se trouvent, le type d'interface de dialogue, les possibilités de combat, le type d'univers dans lequel l'histoire prend place, etc. L'amateur peut alors puiser de l'inspiration dans cet ensemble disparate d'éléments qui l'habite et qui constitue son répertoire personnel, ici moins instrumental que culturel. Comme le relève Jacques Siron, « improviser suppose qu'on choisit entre plusieurs possibilités, et qu'on développe une pensée stratégique à partir d'un matériel ${ }^{31} »$. L'improvisateur fait continuellement face à l'incertitude. Après avoir réalisé un premier jet, le questionnement se renouvelle : et maintenant, que faire? Nous en arrivons à l'élément central qui permet de saisir le sens de l'improvisation telle qu'elle est pratiquée : elle est affaire de possibilités, de choix et de décisions. Au début, «c'est un nouveau projet, il y a encore rien de fait » explique Hector, qui apprécie particulièrement cette étape :

J'aime beaucoup [...] poser les structures, etc. Puis il y a une partie [où] on voit que le jeu commence à fonctionner, ça c'est super fun franchement, c'est une de mes parties préférées. C'est vraiment le début du développement, mais on a déjà mis quelques bases on sait déjà cliquer on a déjà quelques interactions, bon il y a pas les animations il y a pas tout ça, on affiche en brut ce qui vient de se passer mais on voit que ça fonctionne, ça c'est déjà super sympa.

Pour «voir que le jeu commence à fonctionner ", Hector est passé par une phase de test : il a essayé lui-même son début de jeu, il a manipulé l'avatar et a vérifié que les interactions programmées avec d'autres éléments en cours de création avaient bien un comportement appréciable. Il ne s'agit pas de vérifier que l'œuvre corresponde à un game design document ${ }^{32}$ préétabli, mais bien d'éprouver si les éléments ajoutés intuitivement produisent leurs effets. Baptiste explique d'ailleurs que c'est bien l'absence de cahier des charges (des «standards ») qui permet de libérer l'appréciation de toute forme de pression; ce qu'il n'est plus en mesure de vivre depuis que son précédent jeu a connu un succès accidentel : 
Vu qu'il y [avait] pas de, de pallier [d'appréciation] où je me dis « ah zut, c'est pas comme dans mes rêves » et ben c'est pas grave [...] il y [avait] pas de déception à la sortie alors que du coup après, vu que [nom de jeu, anonymisé] a marché, maintenant quand je me dis [que je pourrais créer un nouveau jeu] ben forcément je me dis « hé faudrait vraiment que ce soit vraiment pas mal quoi » et donc après c'est très vite décevant ce que je fais.

Le sentiment de liberté est particulièrement fort au début d'un projet : il n'y a encore aucune contrainte, les problèmes techniques ne s'imposent pas à l'instrumentiste et les bases du jeu sont seulement en train d'être posées. Au fur et à mesure de la réalisation $\mathrm{du}$ jeu, ce sentiment de liberté sera entretenu par les acteurs et constituera un prérequis à l'improvisation. Plus fondamentalement encore, être libre, c'est aussi être libre d'arrêter la création à tout moment. Aussi, Hector " préfère être tout seul » :

Si jamais dans deux heures [...] j'ai plus envie de faire mon projet, ben je peux le planter là et je plante personne quoi je veux dire. J'ennuie personne, ça fout personne dans la merde, il y a pas quelqu'un qui va être déçu, qui va râler, je suis un peu libre quoi (Hector).

La liberté est nécessaire à l'improvisateur parce qu'il lui faut agir par appréciation lors du corps-à-corps avec l'œuvre : l'amateur-créateur se fait amateur-joueur et cherche à vérifier si les effets de goûts attendus sont effectivement produits. Deux résultats peuvent alors survenir : soit l'informateur « accroche » (Hector) et continue sa création (il relance son improvisation), soit il décroche et, n'ayant pas obtenu satisfaction, il arrête son projet. En jouant à son propre jeu, l'amateur cherche à développer des prises sur son œuvre et il vérifie qu'elle lui plaît.

Tentons de répondre, pour conclure, à la question que pose cette partie : où se situe la part de jeu dans la création de nos informateurs? Les amateurs, en accordant une place centrale au plaisir de création suscité par la gestion des incertitudes qui se présentent à eux pendant la réalisation, introduisent du jeu dans leur régime d'expérience créative. La création étant essentiellement une manière de provoquer des sensations pour euxmêmes (ce qui exige une attitude ouverte à l'inattendu), leur pratique passe alors nécessairement par une part d'improvisation. L'improvisation vidéoludique, par le caractère interactif du média, demande à l'amateur d'adopter un processus itératif dans lequel il se retrouve alternativement créateur et joueur. C'est en jouant à sa propre création que l'improvisation peut se renouveler, être relancée, prendre de nouvelles directions, modifier la suite du projet, etc. Cela a pour effet de couper momentanément le geste créatif, mais aussi de le nourrir. L'improvisation vidéoludique est structurée par un rythme en deux temps: elle repose sur des modifications et améliorations successives du jeu. Elle est itérative et c'est précisément dans cette dynamique-là que se loge le jeu créatif : l'auteur des gestes n'est en réalité pas tout à fait certain de ce qu'il est en train de faire, il ne sait pas exactement ce sur quoi sa démarche va déboucher. Il y a une part de surprise et d'inattendu, avec laquelle le créateur prend plaisir à jouer.

\section{Conclusions}

$\mathrm{Au}$ terme de cet article, nous sommes donc en mesure de proposer une définition certes fragmentaire, partiale, mais revendiquée comme telle - de ce que signifie "créer» dans la pratique ludique. Si l'on s'en tient à la strate pragmatique de l'expérience des acteurs, créer consiste en l'agencement (et la production éventuelle) 
de matériaux issus d'un répertoire. Quand la création est abordée comme un jeu, ou, tout du moins, lorsqu'elle est envisagée comme source de plaisir ludique, elle passe aussi par l'improvisation: le créateur s'engage dans une gestion de l'incertitude liée à l'assemblage satisfaisant de matériaux. Si l'on renverse la perspective, que signifie « jouer » au regard de l'expérience de création? Jouer, dans sa dimension pragmatique, nécessite toujours au moins d'inventer ou de réinventer dans l'instant des manières de faire. Le joueur dispose d'éléments et de modalités de prises, qu'il participe à forger, et agence, dans la mesure de ses possibilités, ces éléments pour obtenir satisfaction introduisant ainsi une part de créativité dans son dispositif ludique. Les activités de jeu et de création (tant du côté des joueurs que des créateurs amateurs) ne peuvent dès lors être isolées : elles s'entrecroisent et se nourrissent tout au long de la pratique.

La mise en dialogue de nos terrains d'enquête permet donc d'abord de les éclairer mutuellement : il y a bien du jeu dans la création de jeux vidéo en amateur et de la création chez les joueurs assidus. Or, cela rend également évident le potentiel heuristique et central de la notion d'improvisation dans l'analyse des pratiques ordinaires de création et de jeu. Cette notion ouvre en effet la voie à la compréhension du jeu de la création: quand elle se déroule sans plan préétabli, la création est une tentative dans l'instant et non l'exécution d'une liste de tâches. L'improvisation permet aussi de saisir la part créative de tout jeu : il n'y a pas de jouer sans errance, sans choix, sans tentative - sans improvisation. Cette dernière ne peut, comme dans le cas d'autres pratiques culturelles, reposer que sur un répertoire évolutif, acquis, incorporé, que le joueur peut mobiliser.

Improviser, c'est enfin une activité dans laquelle l'improvisateur se place comme premier joueur, presque comme un médiateur du jeu vers le public: toute improvisation peut être spectacularisée, mais elle est d'abord affaire de relation intime entre l'acteur et son instrument (son logiciel, sa manette, son jeu). L'intimité de cette relation constitue d'ailleurs la dernière caractéristique commune à ces deux expériences (et au regard de laquelle l'adoption d'une méthode commune aux deux enquêtes de terrain est pertinente) : elle souligne, par les liens singuliers qu'elle tisse entre le joueur, l'objet-jeu et sa pratique, la nécessaire part de création inhérente à toute expérience ludique.

\section{BIBLIOGRAPHIE}

Aarseth Espen, « Computer game studies, year one (editorial) », Game Studies, vol. 1, $\mathrm{n}^{\circ}$ 1, 2001.

URL : http://www.gamestudies.org/0101/editorial.html [consulté le 24/03/2021]

Auray Nicolas, « L'engagement des joueurs en ligne. Ethnographie d'une sociabilité distanciée et restreinte ", Cahiers du Numérique [en ligne], vol. 4, no 2, 2003, p. 83-100. URL : https:// www.cairn.info/revue-les-cahiers-du-numerique-2003-2-page-83.htm [consulté le 24/03/2021]

Barnabé Fanny, « Rhétorique du détournement vidéoludique. Le cas de Pokémon », thèse de doctorat en arts et sciences humaines, sous la direction de Jean-Pierre Bertrand et Björn- 
Olav Dozo, Université de Liège, 2017. URL : https://orbi.uliege.be/handle/2268/210764 [consulté le $24 / 03 / 2021]$

Bessy Christian et Chateauraynaud Francis, Experts et faussaires. Pour une sociologie de la perception, Paris, Métailié, coll. « Leçons de choses », 1995.

Bonenfant Maude, «La conception de la "distance" de Jacques Henriot : un espace virtuel de jeu », Sciences du jeu, no 1, 2013.

Bonenfant Maude, Le Libre Jeu. Réflexion sur l'appropriation de l'activité ludique, Montréal, Liber, 2015.

Boutet Manuel, «Jouer aux jeux vidéo avec style. Pour une ethnographie des sociabilités vidéoludiques », Réseaux [en ligne], vol. 3-4, no 173-174, 2012, p. 207-234.

Crawford Garry, "Forget the magic circle (or towards a sociology of video games) ", in Proceedings of the Under the Mask 2 Conference, University of Bedfordshire, 5 juin 2009. URL : http://usir.salford.ac.uk/id/eprint/19350/ [consulté le 24/03/2021]

Delbouille Julie, « Négocier avec une entité jouable : les processus d'appropriation et de distanciation entre joueur, avatars et personnages vidéoludiques ", thèse de doctorat en sciences sociales et comportementales, sous la direction de Björn-Olav Dozo et Christine Servais, Université de Liège, 2019. URL : https://orbi.uliege.be/handle/2268/240605 [consulté le 24/03/2021]

Dozo Björn-Olav et Krywicki Boris, « "Beaux livres” sur les jeux vidéo et presse vidéoludique : transferts et transformations du capital ludique ", in Actes du colloque Penser (avec) la culture vidéoludique, Université de Lausanne, 5-7 octobre 2017.

Frasca Gonzalo, «Ludologists love stories, too: notes from a debate that never took place », in Marinka Copier et Joost Raessens (dir.), Proceedings of the 2003 DiGRA International Conference, Université d'Utrecht, 4-6 novembre, s.l., Digital Games Research Association, vol. 2, 2003. URL : http://www.digra.org/wp-content/uploads/digital-library/05163.01125.pdf [consulté le 24/03/2021]

Grandjean Guillaume, «Quel lieu pour l'exploration? Approche formelle d'une incertitude spatialisée ", in Actes du colloque Entre le jeu et le joueur : écarts et médiations, Université de Liège, 25-27 octobre 2018.

Hennion Antoine, « Réflexivités. L'activité de l'amateur » [en ligne], Réseaux, vol. 1, no 153, 2009, p. 55-78.

Henriot Jacques, Le Jeu, Paris, Presses universitaires de France, coll. « Sup. Initiation philosophique », 1969.

Henriot Jacques, Sous couleur de jouer. La métaphore ludique, Paris, José Corti, 1989.

Hurel Pierre-Yves, «L'expérience de création de jeux vidéo en amateur. Travailler son goût pour l'incertitude ", thèse de doctorat en sciences sociales et comportementales, sous la direction de Christine Servais, Université de Liège, 2020. URL : https://orbi.uliege.be/handle/2268/247377 [consulté le 24/03/2021]

Hurel Pierre-Yves, «Le passage du jeu à la création : le cas du jeu vidéo amateur », Sciences du jeu, $\mathrm{n}^{\mathrm{o}}$ 7, 2017.

Juul Jesper, «The repeatedly lost art of studying games ", Game Studies, vol. 1, n 1, 2001. URL : http://www.gamestudies.org/0101/juul-review/ [consulté le 24/03/2021] 
Kaufmann Jean-Claude, L'Entretien compréhensif, Paris, Armand Colin, coll. « 128. Sociologie, anthropologie. L'Enquête et ses méthodes », 2011.

Laurel Brenda, Computers as theatre, Reading, Addison-Wesley Longman, 1993.

Lejeune Christophe, Manuel d'analyse qualitative. Analyser sans compter ni classer, Louvain-la-Neuve, De Boeck, coll. « Méthodes en sciences humaines », 2014.

Margel Serge, «De l'improvisation. Étude lexicale d'une notion », in Serge Margel (dir.), Pratiques de l'improvisation. Journées d'études à la Manufacture des 5 et 6 juin 2015, Lausanne, BSN Press, coll. « A contrario Campus ", 2016, p. 9-19.

Murray Janet, Hamlet on the Holodeck. The Future of Narrative in Cyberspace, New York, Free Press, 1997.

Rueff Julien, « Où en sont les "game studies” ? " [en ligne], Réseaux, vol. 5, no 151, 2008, p. 139-166. URL : https://www.cairn.info/revue-reseaux1-2008-5-page-139.htm [consulté le 24/03/2021]

Ryan Marie-Laure, « Computer games as narrative », in Avatars of Story, Minneapolis, University of Minnesota Press, 2006, p. 181-203.

Siron Jacques, La Partition intérieure : jazz, musiques improvisées, Paris, Outre Mesure, coll. « Théories », 1992.

Taylor T. L., Play between worlds. Exploring online game culture, Cambridge, The MIT Press, 2006.

Zabban Vinciane, « Retour sur les game studies. Comprendre et dépasser les approches formelles et culturelles du jeu vidéo » [en ligne], Réseaux, vol. 3-4, no 173-174, 2012, p. 137-176.

Winnicott Donald, Jeu et réalité, l'espace potentiel, Paris, Gallimard, 1975.

\section{NOTES}

1. Julien Rueff, «Où en sont les "game studies” ? [en ligne], Réseaux, vol.5, no 151, 2008, p. 139-166. URL: https://www.cairn.info/revue-reseaux1-2008-5-page-139.htm [consulté le 24/03/2021]

2. Vinciane Zabban, «Retour sur les game studies. Comprendre et dépasser les approches formelles et culturelles du jeu vidéo », Réseaux, vol. 3-4, no 173-174, 2012, p. 137-176.

3. Brenda Laurel, Computers as theatre, Reading, Addison-Wesley Longman, 1993 ; Janet Murray, Hamlet on the Holodeck. The Future of Narrative in Cyberspace, New York, Free Press, 1997 ; MarieLaure Ryan, "Computer games as narrative ", in Avatars of Story, Minneapolis, University of Minnesota Press, 2006, p. 181-203.

4. Espen Aarseth, « Computer game studies, year one (editorial) », Game Studies, vol. 1, n 1, 2001. URL : http://www.gamestudies.org/0101/editorial.html [consulté le 24/03/2021] ; Jesper Juul, "The repeatedly lost art of studying games", Game Studies, vol. 1, n ${ }^{\circ} 1,2001$. URL: http:// www.gamestudies.org/0101/juul-review/ [consulté le 24/03/2021] ; Gonzalo Frasca, « Ludologists love stories, too: notes from a debate that never took place », in Marinka Copier et Joost Raessens (dir.), Proceedings of the 2003 DiGRA International Conference, Université d'Utrecht, 4-6 novembre, s.l., Digital Games Research Association, vol. 2, 2003. URL : http://www.digra.org/wp-content/ uploads/digital-library/05163.01125.pdf [consulté le 24/03/2021]

5. Nicolas Auray, «L'engagement des joueurs en ligne. Ethnographie d'une sociabilité distanciée et restreinte ", Cahiers du Numérique [en ligne], vol. 4, no 2, 2003, p. 83-100. URL: https:// www.cairn.info/revue-les-cahiers-du-numerique-2003-2-page-83.htm [consulté le 24/03/2021]; 
T. L. Taylor, Play between worlds. Exploring online game culture, Cambridge, The MIT Press, 2006 ; Garry Crawford, "Forget the magic circle (or towards a sociology of video games) ", in Proceedings of the Under the Mask 2 Conference, University of Bedfordshire, 5 juin 2009. URL : http://usir.salford.ac.uk/id/eprint/19350/ [consulté le 24/03/2021]

6. Julie Delbouille, «Négocier avec une entité jouable: les processus d'appropriation et de distanciation entre joueur, avatars et personnages vidéoludiques ", thèse de doctorat en sciences sociales et comportementales, sous la direction de Björn-Olav Dozo et Christine Servais, Université de Liège, 2019. URL: https://orbi.uliege.be/handle/2268/240605 [ consulté le 24/03/2021]

7. Pierre-Yves Hurel, «L'expérience de création de jeux vidéo en amateur. Travailler son goût pour l'incertitude ", thèse de doctorat en sciences sociales et comportementales, sous la direction de Christine Servais, Université de Liège, 2020. URL : https://orbi.uliege.be/handle/2268/247377 [consulté le 24/03/2021]

8. Jean-Claude Kaufmann, L'Entretien compréhensif, Paris, Armand Colin, coll. «128. Sociologie, anthropologie. L'Enquête et ses méthodes ", 2011.

9. Christophe Lejeune, Manuel d'analyse qualitative. Analyser sans compter ni classer, Louvain-laNeuve, De Boeck, coll. « Méthodes en sciences humaines », 2014.

10. Par souci d'anonymisation, tous les prénoms des informateurs ont été modifiés dans les deux enquêtes.

11. Le terme " informateur » est employé dans ce texte pour désigner les personnes interrogées au cours de nos recherches (joueurs et créateurs amateurs). Le choix de ce vocable, au détriment de celui d' "enquêté ", vise à refléter au mieux notre inscription en recherche qualitative : il s'agit de considérer ces personnes comme étant en capacité d'expliquer leur vécu.

12. Serge Margel, «De l'improvisation. Étude lexicale d'une notion », in Serge Margel (dir.), Pratiques de l'improvisation. Journées d'études à la Manufacture des 5 et 6 juin 2015, Lausanne, BSN Press, coll. « A contrario Campus », 2016, p. 11.

13. Ibid., p. 9.

14. Donald Winnicott, Jeu et réalité, l'espace potentiel, Paris, Gallimard, 1975, p. 75.

15. Christian Bessy et Francis Chateauraynaud, Experts et faussaires. Pour une sociologie de la perception, Paris, Métailié, coll. « Leçons de choses », 1995, p. 235.

16. Ibid., p. 245.

17. Björn-Olav Dozo et Boris Krywicki, « "Beaux livres” sur les jeux vidéo et presse vidéoludique : transferts et transformations du capital ludique », in Actes du colloque Penser (avec) la culture vidéoludique, Université de Lausanne, 5-7 octobre 2017.

18. Ces derniers viennent potentiellement «transgresser » le schéma corporel initial du joueur, comme nous l'avons montré dans notre thèse de doctorat (Delbouille, op. cit.)

19. Guillaume Grandjean, «Quel lieu pour l'exploration? Approche formelle d'une incertitude spatialisée ", in Actes du colloque Entre le jeu et le joueur : écarts et médiations, Université de Liège, 25-27 octobre 2018.

20. Jacques Henriot, Le Jeu, Paris, Presses universitaires de France, coll. «Sup. Initiation philosophique », 1969, p. 76.

21. Maude Bonenfant, «La conception de la "distance" de Jacques Henriot : un espace virtuel de jeu ", Sciences du jeu, no 1, 2013, p. 1.

22. Jacques Henriot, Sous couleur de jouer. La métaphore ludique, Paris, José Corti, 1989, p. 252.

23. Voir notamment les travaux de Manuel Boutet, «Jouer aux jeux vidéo avec style. Pour une ethnographie des sociabilités vidéoludiques ", Réseaux [en ligne], vol.3-4, $n^{\circ}$ 173-174, 2012, p. 207-234. 
24. Dont certaines tentent de sortir du cadre du play prévu et préconisé par le jeu, comme dans le cas de l'exploitation de glitchs [failles techniques] dans la réalisation d'un speedrun [finir un jeu le plus vite possible]; voir notamment Fanny Barnabé, «Rhétorique du détournement vidéoludique. Le cas de Pokémon", thèse de doctorat en arts et sciences humaines, sous la direction de Jean-Pierre Bertrand et Björn-Olav Dozo, Université de Liège, 2017.

25. Pierre-Yves Hurel, « Le passage du jeu à la création : le cas du jeu vidéo amateur », Sciences du jeu, $\mathrm{n}^{\circ} 7,2017$.

26. Pierre-Yves Hurel, «L'expérience de création de jeux vidéo en amateur. Travailler son goût pour l'incertitude ", op. cit.

27. En effet, jusqu'à récemment, les jeux RPG Maker se partageaient surtout sous un format qui les rendait non seulement jouables en tant que jeux réalisés, mais aussi consultables et modifiables via le logiciel. Tout le monde pouvait ouvrir les fichiers du jeu pour comprendre comment celui-ci était conçu (cette modalité de partage est possible dans une majorité d'outils de création).

28. role-playing game (littéralement « jeu de rôle »)

29. Nous nous concentrons ici sur des mécaniques ludiques, car elles font la spécificité du média, mais le même phénomène peut s'observer chez les informateurs sur les aspects graphiques, scénaristiques ou sonores.

30. Jacques Siron, La Partition intérieure: jazz, musiques improvisées, Paris, Outre Mesure, coll. « Théories ", 1992, p. 88.

31. Ibid., p. 79.

32. Lorsqu'un studio professionnel de développement de jeux vidéo initie un nouveau projet, il met au point un game design document qui liste tous les éléments à développer pour réaliser le projet (éléments graphiques et sonores, système de jeu et des interfaces, etc.). Ce document sert ensuite de référence à toutes les personnes impliquées dans la réalisation du projet.

\section{RÉSUMÉS}

Jouer et créer sont deux activités qui semblent liées : il y a une part de créativité dans l'acte de jeu et une part de jeu dans l'acte créatif. Il n'est cependant pas aisé de caractériser, au-delà de cette intuition, ce qui unit ces deux activités. Nous proposons d'éclairer ce lien par la notion d'improvisation, que nous allons construire depuis deux perspectives pragmatiques : d'abord en identifiant le caractère improvisé de l'acte de jouer aux jeux vidéo et ensuite en cherchant à comprendre ce qu'il y a d'improvisé dans la création de jeux vidéo amateurs.

Playing and creating are two activities which seem to be connected: creativity is a part of playing as well as there is playfulness in the creative process. However, it is not easy to grasp what unites, beyond this intuition, these two activities. This paper aims to shed some light on this connection through the notion of improvisation, which will draw upon two pragmatic perspectives: first by identifying the improvised nature of the act of playing video games, and second by seeking to understand what is improvised in the creation of amateur video games. 
INDEX

Mots-clés : jouer, créer, improviser, jeu vidéo, amateurs

\section{AUTEURS}

\section{JULIE DELBOUILLE}

Julie Delbouille est docteure en information et communication et a réalisé sa thèse à l'université de Liège. Après une formation en histoire de l'art, elle se spécialise en études de réception en étudiant les discours sur le jeu vidéo dans l'espace belge francophone. Ses recherches actuelles se focalisent sur la relation entre joueur, personnage et avatar vidéoludique, sur l'immersion, ainsi que sur les pratiques de jeu secondaire. Elle est membre du Liège game lab (LGL) et du Laboratoire d'étude sur les médias et la médiation (Lemme)de l'université de Liège, place du 20Août, 7, 4000 Liège ; jdelbouille@alumni.uliege.be

\section{PIERRE-YVES HUREL}

Pierre-Yves Hurel est docteur en information et communication et assistant au département Médias, culture et communication à l'université de Liège. Il a consacré sa thèse doctorale à l'étude de la création de jeux vidéo en amateur, analysant les dynamiques de création à l'œuvre dans le cadre de l'utilisation de logiciels de facilitation tels que RPG Maker ou Construct 3. Il est membre du Liège game lab (LGL) et du Laboratoire d'étude sur les médias et la médiation de (Lemme)de l'université de Liège, place du 20-Août, 7, 4000 Liège ; pyhurel@uliege.be 